\title{
Herniation of ureter: a rare cause of hydroureteronephrosis
}

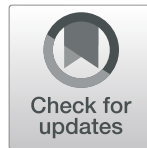

Preeti Gupta ${ }^{1 *}$, Pankaj Sharma', Vinay Maurya ${ }^{2}$ and Mukul Bhatia ${ }^{2}$

\begin{abstract}
Background: Omentum and bowel loops are commonly seen in inguinal hernia. An unusual case of extraperitoneal herniation of ureter was detected in hernial sac with other contents, on computed tomography (CT) urography.

Case presentation: A 54-year-old male presented with inguinal hernia, with no previous history of surgery or trauma. Pre-operative screening showed a unilateral hydroureteronephrosis involving the pelvicalyceal system and entire ureter. No calculus was visualized in the ureter on ultrasound. On non-contrast CT (NCCT) followed by CT urography, the ureter was seen descending into the hernia sac causing hydroureteronephrosis. The patient subsequently underwent mesh repair surgery of the inguinal hernia with meticulous reduction of the herniated contents including the ureter, resulting in complete resolution of the hydroureteronephrosis.

Conclusion: An isolated extraperitoneal inguinal herniation of the ureter without concomitant urinary bladder involvement is extremely rare, especially with no prior history of trauma or surgery, as seen in our case. The case also exemplifies the role of pre-operative imaging as a protocol in cases of inguinal hernia to prevent per-operative complications.
\end{abstract}

Keywords: Ureter, Inguinal, Hernia, CT urography

\section{Background}

Inguinal hernia is one of the commonest elective surgeries performed worldwide [1]. Variety of hernia sac contents has been described in the literature. The commonest among these are omentum and bowel loops $[1,2]$. We report a case of herniation of the native ureter in to the inguinal canal. Isolated inguinal herniation of the ureter is extremely rare, which if left undiagnosed can cause operative complications in an otherwise routine hernia repair surgery.

\section{Case presentation}

\section{Clinical presentation}

A 54-year-old male was admitted to our tertiary care hospital with left-sided inguinal hernia. The patient

\footnotetext{
* Correspondence: preeti.preetigupta@gmail.com

${ }^{1}$ Department of Radiodiagnosis and Imaging, Command Hospital (Eastern

Command), Kolkata, West Bengal, India

Full list of author information is available at the end of the article
}

presented with swelling in left inguinal region along with dull dragging pain. There was no history of surgery or trauma. On clinical examination, the hernia was found to be of direct type. The contents were irreducible. Elective surgery was planned and pre-op evaluation was done, including abdominal ultrasound. Pre-operative ultrasound is a routine procedure at our center to rule out underlying mass leading to herniation of contents through a potentially weakened anterior abdominal wall.

\section{Radiological findings}

The patient reported to the radiology department for abdominal ultrasound. On ultrasound (Fig. 1a), the left pelvicalyceal system was found dilated along with the ureter along its entire length. No calculi could be identified. No hydroureteronephrosis was seen on the right side. Patient did not give any history of urolithiasis in the past or passage of stone recently. Urinary bladder and bilateral vesicoureteral junctions were normal. No 


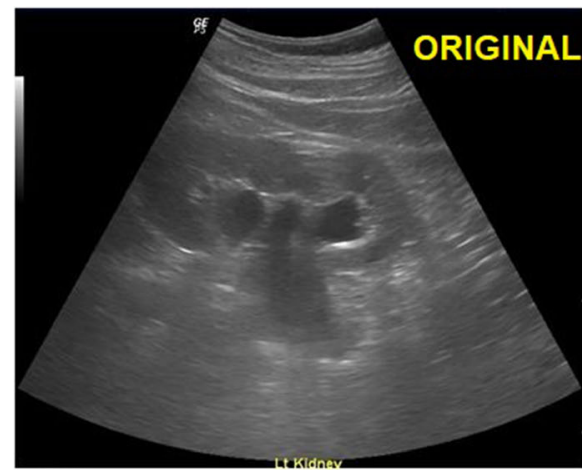

(a)

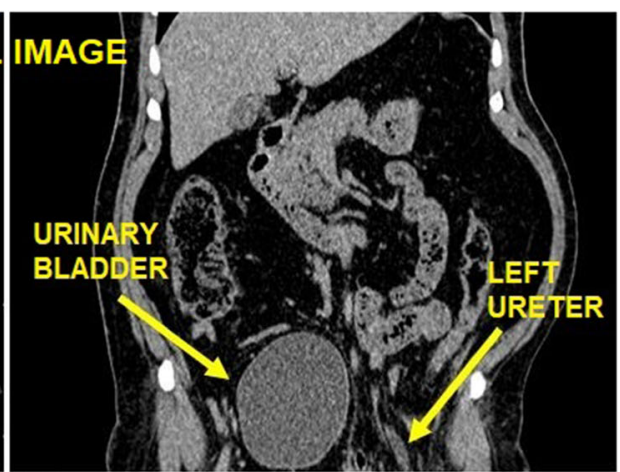

(b)

Fig. 1 a Left hydroureteronephrosis without any calculus on ultrasound. b Coronal NCCT image with dilated left ureter entering the inguinal hernia sac

other abnormality was detected in ultrasonographic examination. His renal function tests were also unremarkable. Following this, patient was sent for noncontrast computed tomography (NCCT) scan of kidney, ureter, and bladder area. On CT scan, no stone was detected. The left pelvicalyceal system and ureter were seen dilated with the distal ureter extending into the herniated sac (Fig. 1b). A CT urography was performed to confirm the findings of NCCT. The hernia sac contained bowel loops, omental fat, and contrast opacified ureter well identified in the pyelographic phase in axial and coronal images respectively (Fig. 2). No evidence of obstruction or incarceration was seen. The urinary bladder was seen in its normal anatomical location. Volume rendered image is also provided which confirms the same (Fig. 3) and shows the normally opacified rightsided pelvicalyceal system.

\section{Operative findings}

Thereafter, the patient was operated with careful dissection of the ureter. Mesh repair was done. There were no unanticipated events during the surgery. On follow-up, there was complete resolution of the left-sided hydroureteronephrosis for which patient had been asymptomatic all this while. The post-op site healed with no recurrence of the swelling in the inguinal region.

\section{Discussion}

The commonest contents of the hernia sac are omental fat and bowel loops [1,2]. Extensive review of literature showed herniation of the ureter as extremely uncommon and only 140 cases have been reported so far [3]. Vermiform appendix, urinary bladder, ovary, and fallopian tubes have also been reported rarely as part of the herniated sac $[1,2]$. As per the literature, appendix was found in $0.1 \%$ of groin hernial sacs [2]. Reports of tubo-ovarian herniation have been seen in $2.9 \%$ hernia cases and urinary bladder herniation in $0.36 \%$ cases [2].

Herniated ureter is mostly seen following trauma or adhesions caused by iatrogenic injury, for example, in cases of transplanted kidney [3, 4]. Cases also have been reported following radiotherapy [5]. Herniation of native

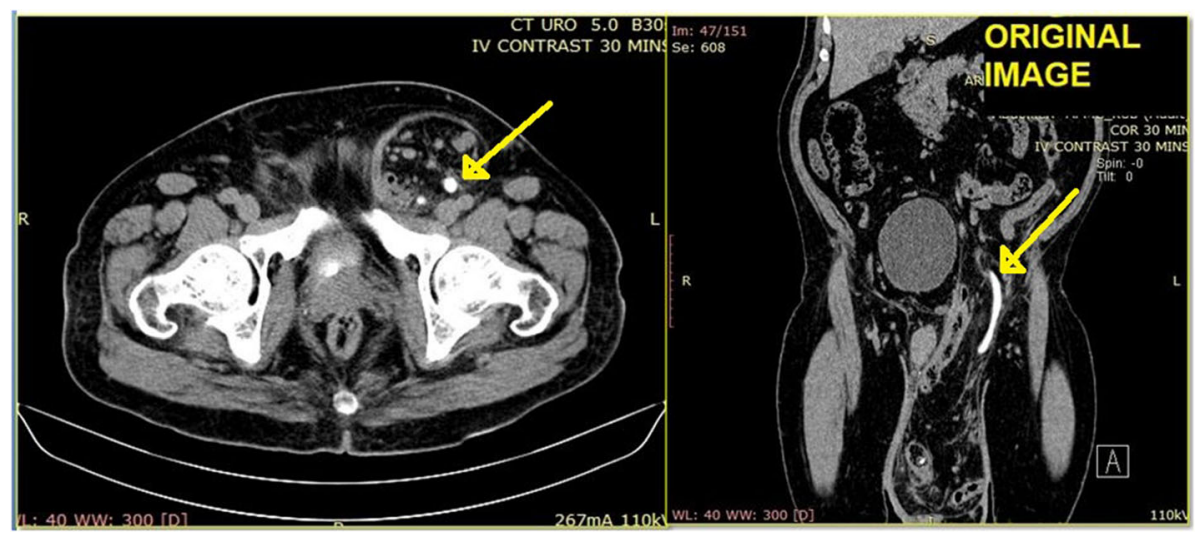

Fig. 2 CT urography shows hernia sac in left inguinal region with opacified ureter, in axial and coronal sections 


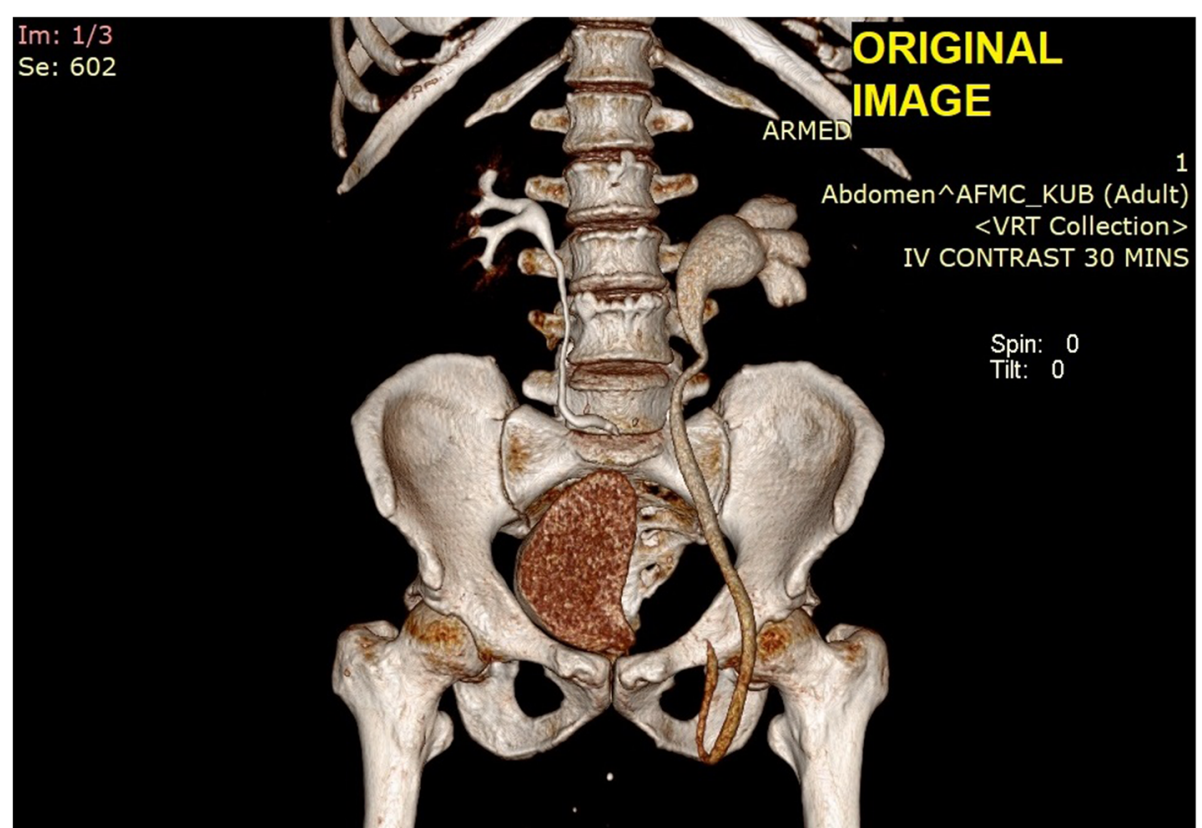

Fig. 3 Volume rendered image (VRI) of the left inguinal hernia sac with left ureter inside, in the pyelographic phase

ureter without any associated history of injury or surgery is not well documented. In the present case, there was no such history, and that makes it extremely unique. There are case reports of herniation of the ureter along with the urinary bladder [6]; however, in this case, it was an isolated herniation of the ureter with hydroureteronephrosis without a visible calculus. Two types of ureteral herniations have been described: paraperitoneal and extraperitoneal $[7,8]$. Paraperitoneal herniation of the ureter is when the ureter is adherent to the posterior peritoneum and it slides into the inguinal canal. It is more likely to be acquired inguinal herniation of the ureter. In extraperitoneal herniation, the ureter is congenitally fused with the Wolffian duct and is drawn into the inguinal canal. Presence of primordial germ cells in close proximity to the pro-, meso-, and metanephros during the embryological development may lead to this fusion in extraperitoneal subtype leading to herniation of the ureter into the inguinal canal. This case belongs to extraperitoneal category though there was no visible ureteric calculus which is found to be associated with this type of herniation of ureter [5].

\section{Conclusions}

This case brings to light a rare cause of hydroureteronephrosis and reiterates the importance of pre-operative imaging as a protocol to look for underlying unusual contents and prevent potential complications during the surgery. It also highlights the role of CT scan as a problem-solving tool in atypical cases.

\section{Abbreviations}

CT: Computed tomography; NCCT: Non-contrast computed tomography; VRI: Volume rendered image

\section{Acknowledgements}

Not applicable

\section{Authors' contributions}

PG and MB performed the patient's ultrasound and were involved in detailed history taking. PG, PS, and VM performed the patient's non-contrast $C T$ scan and the CT urography. All the four listed authors have been involved in drafting the paper and revising it critically for important intellectual content, and all were involved in the final approval of the version to be published. All the authors agree to be held accountable for all aspects of the work in ensuring that questions related to the accuracy or integrity of any part of the work are appropriately investigated and resolved. The authors read and approved the final manuscript.

\section{Funding}

Not applicable

\section{Availability of data and materials}

The datasets used and/or analyzed during the current study are available from the corresponding author on reasonable request.

\section{Declarations}

Ethics approval and consent to participate

The inclusion of patient data was done only after acquiring approval on the Institutional Ethical Committee of Command Hospital, Kolkata.

\section{Consent for publication}

Written and informed consent was obtained from the patient before use of the data pertaining to the patient in the study.

Competing interests

The authors declare that they have no competing interests. 


\section{Author details}

'Department of Radiodiagnosis and Imaging, Command Hospital (Eastern Command), Kolkata, West Bengal, India. ${ }^{2}$ Department of Radiodiagnosis and Imaging, Base Hospital Delhi Cantt, New Delhi, India.

Received: 18 November 2020 Accepted: 9 June 2021

Published online: 22 July 2021

\section{References}

1. S. Shrivastva M, Verma RK, Goyal S (2015) "Uncommon contents of inguinal hernial sac": a surgical dilemma. Indian J Surg 77(Suppl 2):305-309. https:// doi.org/10.1007/s12262-013-0806-7

2. Gurer A, Ozdogan M, Ozlem N, Yildirim A, Kulacoglu H, Aydin R (2006) Uncommon content in groin hernia sac. Hernia. 10(2):152-155. https://doi. org/10.1007/s10029-005-0036-4

3. Odisho A et al (2010) Inguinal herniation of a transplant ureter. Kidney Int 78:115 PMID: 20551935

4. Azhar R, Boutros M, Hassanain M, Polyhronopoulos G, Chaudhury P, Tchervenkov J, Cabrera T (2009) A rare case of obstructive uropathy in renal transplantation: ipsilateral indirect inguinal herniation of a transplant ureter. Transplantation. 88(8):1038-1039. https://doi.org/10.1097/TP.0b013e3181 b9e28d

5. Prete FP, Pezzolla A, De Leo V, Di Palma G, Prete F (2016) Inguino-scrotal herniation of the ureter containing stones. Hernia. 20(6):887-890. https://doi. org/10.1007/s10029-015-1400-7

6. De Angelis M, Mantovani G, Di Lecce F, Boccia L (2018) Inguinal bladder and ureter hernia Permagna: definition of a rare clinical entity and case report. Case Rep Surg 2018:9705728Published 2018 Sep 30. https://doi.org/1 $0.1155 / 2018 / 9705728$

7. ZarraonandiaAndraca A et al (2009) Inguinal ureteral hernia: a clinical case. Arch Esp Urol 62(9):755-757 PMID: 19959862

8. Lu A, Burstein J (2012) Paraperitoneal inguinal hernia of ureter. J Radiol Case Rep 6(8):22-26. https://doi.org/10.3941/jrcr.v6i8.1027

\section{Publisher's Note}

Springer Nature remains neutral with regard to jurisdictional claims in published maps and institutional affiliations.

\section{Submit your manuscript to a SpringerOpen ${ }^{\circ}$ journal and benefit from:}

- Convenient online submission

- Rigorous peer review

- Open access: articles freely available online

- High visibility within the field

- Retaining the copyright to your article

Submit your next manuscript at $\boldsymbol{\sim}$ springeropen.com 